Pacific Journal of Mathematic 


\title{
HARMONIC ANALYSIS ON COMPACT HYPERGROUPS
}

\author{
RICHARD C. VREM
}

Let $K$ be a compact hypergroup (convo) as defined by R. Jewett. It is shown that Trig $(K)$ is uniformly dense in $C(K)$ and the Peter-Weyl theorem holds. A generalization of the Weil character formula is obtained and a Fourier transform is defined. Analogues of the Riemann-Lebesgue lemma, Parseval's identity and the Riesz-Fischer theorem are proved in this setting. The space $A(K)$ of functions in $L^{1}(K)$ with absolutey convergent Fourier series is shown to be the linear span of the positive-definite functions on $K$ and the equality $A(K)=L^{2}(K) * L^{2}(K)$ is established.

1. Introduction. There has recently been considerable interest shown by some harmonic analysts in the question of which topological spaces have enough structure so that a convolution on the corresponding space of all finite regular Borel measures can be defined. Dunkl [3], Jewett [5] and Spector [10] have all addressed this question and they have given axioms which are essentially the same. Jewett calls these objects convos while Dunkl and Spector refer to them as hypergroups. We shall use the latter terminology but we adopt Jewett's axioms. For a survey of the subject, the interested reader is referred to Ross [8].

This article will be primarily concerned with compact nonabelian hypergroups. In a subsequent paper we will consider lacunarity on compact hypergroups. Throughout this paper $K$ will denote a hypergroup and $M(K)$ the space of finite regular Borel measures on $K$. In $\S 2$ the representation theory of (locally) compact hypergroups is studied. If $K^{\wedge}$ denotes the set of equivalence classes of continuous irreducible representations of $K$ then it is shown that $K^{\wedge}$ separates points of $K$. If $K$ is compact then the elements of $K^{\wedge}$ are finitedimensional and an analogue of the Peter-Weyl theorem is obtained. It is also shown that Trig $(K)$ is uniformly dense in the space $C(K)$ of continuous functions on $K$. $\S 3$ contains basic results regarding the Fourier-Stieltjes transform on $M(K)$. It is also shown that $K^{\wedge}$ will consist of unitary representations precisely when $K$ is a group. The Fourier-Stieltjes series of a regular Borel measure is defined in $\S 4$ and the space $A(K)$ of $L^{1}(K)$ functions with absolutely convergent Fourier series is considered. It is shown that $A(K)$ is the linear span of the positive-definite functions on $K$ and can be written as $L^{2}(K) * L^{2}(K)$ (throughout this paper * will refer to the convolution 
on $M(K)$ ). Finally, we prove $A(K)$ is a regular Banach algebra under convolution and provide an example to show $A(K)$ need not be a Banach algebra under pointwise operations.

The notation used is that of Jewett [5] except $\delta_{x}$ denotes the point mass at $x, x \rightarrow x^{\sim}$ is the involution on $K$ and $I_{A}$ the indicator function of $A$. For each representation $U$ in $K^{\wedge}, H_{U}$ is the corresponding Hilbert space and if $U$ is finite-dimensional $d_{U}$ is the dimension of $U$. If $K$ admits a Haar measure it will be written $m$ and if $K$ is compact then $m$ is assumed to be suitably normalized.

Finally, the author wishes to thank K. A. Ross for his many helpful suggestions and criticisms.

2. Representation theory. We first assume $K$ is an arbitrary locally compact hypergroup. Following Jewett $[5,11.3]$ we define a representation of $K$ as a non norm-increasing *-representation of the Banach *-algebra $M(K)$. The representation will be called continuous if it is weak operator continuous on $M^{+}(K)$ with the cone topology. For notational convenience, we write $U_{x}$ for $U_{i_{x}}$ where $x \in K$. We now give a fundamental example.

EXAMPLE 2.1. Suppose $K$ is a locally compact hypergroup admitting a Haar measure $m$ and let $H=L^{2}(m)$. Jewett [5, 6.2] shows that the left regular representation $T$ of $K$ on $H$ is a faithful representation of $K$. We show that $T$ separates the points of $K$. If $a, b \in K$ with $a \neq b$ then there exist disjoint relatively compact neighborhoods $N_{1}, N_{2}$ of $a^{\smile}$ and $b^{\vee}$ respectively. By [5, 3.2 D] there exist open neighborhoods $W_{1}, W_{2}$ of $e$ so that $\left\{a^{\smile}\right\} * W_{1} \leqq N_{1}$ and $\{b\} * W_{2} \cong N$. It is easy to see that $T_{a}\left(I_{N_{1}}\right)$, is identically 1 on $V=W_{1} \cap W_{2}$ and $T_{b}\left(I_{N_{2}}\right)$ is identically 0 on $V$. Thus $T$ separates points.

The proof of the next theorem is modeled after a proof of Nachbin [7].

THEOREM 2.2. If $U$ is a continuous irreducible representation of a compact hypergroup $K$ then $U$ is finite-dimensional.

Proof. Fix $\zeta, \lambda \in H$ where $H=H_{U}$. Let $\zeta \in H$ and define $T(\zeta)$ as the unique vector in $H$ such that

$$
\langle T \xi, \eta\rangle=\int_{K}\left\langle U_{x} \xi, \zeta\right\rangle\left\langle U_{x} \eta, \lambda\right\rangle^{-} d m(x) \quad \text { for all } \eta \in H .
$$

It is easily shown that $T(\zeta, \lambda)$ is a bounded linear operator on $H$ and that $T(\zeta, \lambda)$ commutes with each $U_{\mu}, \mu \in M(K)$. Thus $T(\zeta, \lambda)$ is scalar, say $T(\zeta, \lambda)=a(\zeta, \lambda) I$. By $[5,7.2 \mathrm{~A}] m=m^{2}$ so that $a(\zeta, \lambda)\langle\xi, \eta\rangle=$ 
$a(\eta, \xi)\langle\zeta, \lambda\rangle$ and hence $a(\zeta, \lambda)=c\langle\zeta, \lambda\rangle^{-}$for some constant $c$. It follows that

$$
\int_{K}\left\langle U_{x} \xi, \zeta\right\rangle\left\langle U_{x} \eta, \lambda\right\rangle^{-} d m(x)=c\langle\xi, \eta\rangle\langle\zeta, \lambda\rangle^{-} .
$$

If we let $\xi=\zeta=\eta=\lambda=\beta$ where $\|\beta\|=1$ then

$$
\int_{K}\left|\left\langle U_{x} \beta, \beta\right\rangle\right|^{2} d m(x)=c .
$$

But the continuous function $x \rightarrow\left|\left\langle U_{x} \beta, \beta\right\rangle\right|^{2}$ has value 1 at $e$ so $c$ is positive.

Let $\left\{\zeta_{i}\right\}_{i=1}^{n}$ be an orthonormal set in $H$. Let $\zeta=\lambda=\zeta_{k}, 1 \leqq k \leqq n$ and $\xi=\eta=\alpha$ in equation (1). Using (1) and the fact that $\left\{\zeta_{k}\right\}$ is an orthonormal set we have

$$
n c=\sum_{k=1}^{n} \int_{K}\left|\left\langle U_{x} \alpha, \zeta_{k}\right\rangle\right|^{2} d m(x) \leqq \int_{K}\left\|U_{x} \alpha\right\|^{2} d m(x) \leqq \int_{K}\left\|U_{x}\right\|^{2}\|\alpha\|^{2} \leqq 1 .
$$

Hence $\operatorname{dim}(H) \leqq c^{-1}$.

We next want to show there are enough continuous irreducible representations of a locally compact hypergroup to separate points. First we require the following lemma.

LEMMA 2.3. Let $K$ be a locally compact hypergroup admitting a Haar measure. Let $T$ be a continuous irreducible *-representation of $M(K)$ on $B(H)$ with $T \mid M_{a}(K) \neq 0$. Then there is a unique continuous irreducible representation $U$ of $K$ such that $U_{\nu}=T_{\nu}$ for all $\nu \in M_{a}(K)$.

Proof. Let $\bar{T}=T \mid M_{a}(K)$. Since $M(K)$ is a Banach *-algebra $[5,6.1 \mathrm{G}]$ we have $\left\|T_{\mu}\right\| \leqq\|\mu\|$ for all $\mu \in M(K)$. Thus $\bar{T}$ is a bounded *-homomorphism. Suppose $\xi \in H$ and $\bar{T}_{\nu}(\xi)=0$ for all $\nu \in M_{a}(K)$ and let $H_{\xi}=\left\{T_{\mu}(\xi): \mu \in M(K)\right\}^{-}$. Since $H_{\xi}$ is a closed $T$-invariant subspace of $H$ we have $H_{\xi}=\{0\}$ or $H_{\xi}=H$. Using the fact that $M_{a}$ is an ideal of $M(K)$ and our hypothesis that $\bar{T} \not \equiv$ one can show $H_{\xi} \neq H$. The irreducibity of $T$ then forces $\xi=0$ and $[5,11.5 \mathrm{~A}]$ gives the existence of a unique representation $U$ of $K$ such that $U_{\nu}=T_{\nu}$ for all $\nu \in M_{a}(K)$. To show $U$ is irreducible, it suffices to show $\bar{T}$ is irreducible. If $X$ is a closed $\bar{T}$-invariant subspace of $H$ then $(\operatorname{span} \bar{T}(X))^{-}$is $T$-invariant since $M_{a}(K)$ is an ideal. If $(\text { span } \bar{T}(X))^{-}=\{0\}$ it follows that $X=0$. Since $(\operatorname{span} \bar{T}(X))^{-} \leqq X$, $(\operatorname{span} \bar{T}(X))^{-}=H$ implies $X=H$.

THEOREM 2.4. Let $K$ be a locally compact hypergroup. There 
are enough continuous irreducible representations of $K$ to separate points.

Proof. By Example 2.1 the regular representation $M(K)$ is faithful so there are enough continuous irreducible *-representations of $M(K)$ to separate points. If $a, b \in K$ with $a \neq b$ then as in Example 2.1 there exists a relatively compact neighborhood $W$ of $e$ so that $\nu=\delta_{a} * L_{W}$ and $\mu=\delta_{b} * L_{W}$ are supported on disjoint sets. So there exists a continuous irreducible *-representation $T$ of $M(K)$ such that $T_{\nu} \neq T_{\mu}$. By Lemma 2.3 there exists a continuous irreducible representation $U$ of $K$ so that $U_{\nu} \neq U_{\mu}$, i.e., $U_{a} \neq U_{b}$.

COROLLARY 2.5. If $K$ is a compact hypergroup then there are enough finite-dimensional continuous irreducible representations of $K$ to separate points.

Proof. This follows from Theorems 2.2 and 2.4.

Unless otherwise stated $K$ will from now on be a compact hypergroup. Suppose $U \in K^{\wedge}$ and $\left\{\zeta_{j}\right\}_{j=1}^{d}$ is $_{\text {in }}$ an orthonormal basis for $H_{U}$. We define coordinate functions for $U$ by $u_{j_{k}}(x)=\left\langle U_{x} \zeta_{k}, \zeta_{j}\right\rangle$ where $1 \leqq j, k \leqq d_{U}$. If $\operatorname{Trig}_{U}(K)$ is the linear span of coordinate functions of $U$ then it is easily seen that $\operatorname{Trig}_{U}(K)$ is independent of the choice of basis for $H_{U}$. Trig $(K)$ will denote the linear span of $U\left\{\operatorname{Trig}_{U}(K): U \in K^{\wedge}\right\}$.

We next establish orthogonality relations for these coordinate functions.

THEOREM 2.6. If $U, V \in K^{\wedge}$ then there exists a constant $k_{U}$ with $k_{U} \geqq d_{U}$ such that

$$
\int_{K} u_{j k}\left(v_{r s}\right)^{-} d m= \begin{cases}k_{U}^{-1} & \text { if } U=V, j=r, k=s \\ 0 & \text { otherwise. }\end{cases}
$$

Moreover, if $K$ is a group then $k_{U}=d_{U}$.

Proof. Suppose $U=V$ and $\left\{\zeta_{j}\right\}_{j=1}^{d}$ is a fixed orthogonal basis for $H_{U}$. Using equation (1) of Theorem 2.2 and the fact that the basis is orthonormal we conclude

$$
\int_{K} u_{j k} \bar{v}_{r s} d m= \begin{cases}c & \text { if } r=j \text { and } k=s \\ 0 & \text { otherwise }\end{cases}
$$

Let $k_{U}=c^{-1}$. Then $d_{U} \leqq k_{U}$ from the last line of the proof of 2.2 and equality occurs when $K$ is a group. 
The case where $U$ and $V$ are not equivalent is handled by a standard argument.

CoRollary 2.7. The dimension of each $\operatorname{Trig}_{U}(K)$ is $d_{U}^{2}$. If fixed coordinate functions are selected for each $U \in K^{\wedge}$ then $\left\{k_{U}^{1 / 2} u_{i j}: U \in K^{\wedge}, 1 \leqq i, j \leqq d_{U}\right\}$ is an orthonormal set in $L^{2}(K)$. Also, $\operatorname{Trig}(K)=\bigoplus\left\{\operatorname{Trig}_{U}(K): U \in K^{\wedge}\right\}$.

LEMMA 2.8. $M(K)$ has a nonnegative approximate unit in $L^{2}(K)$.

Proof. Use normalized indicator functions of neighborhoods of $e$ and $[5,5.1 \mathrm{C}]$.

THEOREM 2.9. Trig $(K)$ is dense in $L^{2}(K)$.

Proof. Let $T$ denote the regular representation of $K$ on $L^{2}(K)$. By $[5,7.2 \mathrm{C}] L^{2}(K)$ is the direct sum of its minimal closed ideals and each of these minimal closed ideals is finite-dimensional. Let $\mu$ be in $M(K)$ and let $\left\{t_{\alpha}\right\}$ be a bounded nonnegative approximate unit as in Lemma 2.8. If $I$ is a minimal closed ideal of $L^{2}(K)$ with $f \in I$, then $\mu * f \in L^{2}(K)$ and hence $t_{\alpha} *(\mu * f) \rightarrow \mu * f$ in $L^{2}(K)$. Since $I$ is closed, we have $\mu * f \in I$, i.e., $I$ is $T$-invariant. Hence $T \mid I$ is a finite-dimensional representation of $K$ which can be written as a direct sum of continuous irreducible subrepresentations, say $L^{2}(K)=$ $\bigoplus\left\{H_{\beta}: \beta \in A\right\}$. Write $T \mid H_{\beta}=T^{\beta}$ and $d(\beta)$ for the dimensional basis for $H_{\beta}$. Suppose $f \in L^{2}(K)$ and $\langle f, g\rangle=0$ for all $g \in \operatorname{Trig}(K)$. Since $T^{\beta} \in K^{\wedge}$ for each $\beta \in A$ we have

$$
0=\int_{K}\left\langle T_{x}^{\beta} g_{j}^{\beta}, g_{i}^{\beta}\right\rangle \bar{f}(x) d m(x)=\left\langle T_{\bar{f}} g_{j}^{\beta}, g_{i}^{\beta}\right\rangle=\left\langle\bar{f} * g_{j}^{\beta}, g_{i}^{\beta}\right\rangle .
$$

Since $\left\{g_{i}^{\beta}: \beta \in A, 1 \leqq i \leqq d(\beta)\right\}$ is a basis for $L^{2}(K)$ we have $\bar{f} * h=0$ for all $h \in L^{2}(K)$. In particular, $\bar{f} * t_{\alpha}=0$ for all $\alpha$ and hence $f=0$.

The following generalization of the Peter-Weyl theorem for compact groups was known to Spector [10, II. 1.3] (compare with $[4,27.40])$.

Corollary 2.10. For $f \in L^{2}(K)$ we have

$$
f=\sum_{U \in K^{\wedge}} \sum_{j, k=1}^{d_{U}} k_{U}\left\langle f, u_{j k}\right\rangle \cdot u_{j k}
$$

where the series is in $L^{2}(K)$. Furthermore, if $\left\{a_{j k}(U): U \in K^{\wedge}, 1 \leqq j\right.$, $\left.k \leqq d_{v}\right\}$ is any set of complex numbers such that 


$$
\sum_{U \in K^{\wedge}} \sum_{j, k=1}^{d_{U}} k_{U}\left|a_{j k}(U)\right|^{2}<\infty
$$

then there is a unique $g \in L^{2}(K)$ such that $\left\langle g, u_{j_{k}}\right\rangle=a_{j_{k}}(U)$ for all $U \in K^{\wedge}, 1 \leqq j, k \leqq d_{U}$ and for which $g=\sum_{U \in K^{\wedge}} \sum_{j, k=1}^{d_{U}} k_{U} a_{j k}(U) u_{j k}$.

Since Trig $(K)$ is not an algebra of functions ([10, II. 1.3]) we cannot apply Stone-Weierstrass. In order to prove Trig $(K)$ is uniformly dense in $C(K)$ we require the following lemmas.

LEMma 2.11. Let $\left\{h_{\alpha}\right\} \subseteq L^{1}(K)^{+}$with $\left\|h_{\alpha}\right\|_{1}=1$ for all $\alpha$. Then $\left\{h_{\alpha}\right\}$ is a left approximate unit in $L^{1}(K)$ if $\lim _{\alpha}\left\|h_{a} I_{K-W}\right\|_{1}=0$ for all neighborhoods $W$ of $e$. Moreover, $h_{\alpha} \rightarrow \delta_{e}$ in the weak-* topology.

Proof. From $[5,5.4 \mathrm{H}]$ we have $\lim _{y \rightarrow e}\left\|f_{y^{\vee}}-f\right\|_{1}=0$ and [5, 3.3B] shows that $\left\|f_{y^{v}}\right\|_{1} \leqq\|f\|_{1}$. The proof that $\left\{h_{\alpha}\right\}$ is a left approximate unit now follows as in [4, 28.52]. A standard argument shows that $h_{\alpha} \rightarrow \delta_{e}$ in the weak-* topology.

LEMMA 2.12. There is a bounded left approximate unit $\left\{h_{\alpha}\right\}$ in $L^{1}(K)$ such that for all $\alpha$ :

(i ) $h_{\alpha} \in$ Trig $^{+}(K)$

(ii) $\left\|h_{\alpha}\right\|_{1}=1$

(iii) $h_{\alpha}$ is a finite sum of functions of the form $g * g^{*}$ where $g \in \operatorname{Trig}(K)$.

Proof. Let $\left\{k_{W}\right\}$ be the approximate unit described in 2.8. Let $\psi_{W}=k_{W} * k_{W}$. The proof now proceeds as in the group case [4, 28.53] (note that this proof does require Corollary 2.10).

The next theorem answers a question of Dunkl [3, 3.7].

THEOREM 2.13. Trig $(K)$ is uniformly dense in $C(K)$.

Proof. Suppose $f \in C(K)$. By Lemma 2.12 there exists a left approximate unit $\left\{t_{\alpha}\right\} \cong \operatorname{Trig}^{+}(K)$ for $L^{1}(K)$. If $h \in L^{1}(K)$ and $f \in C(K)$ it is easy to see that $\|h * f\|_{u} \leqq\|f\|_{u}\|h\|_{1}$ Thus $C(K)$ is a left $L^{1}(K)$-module. By Lemma $2.11 t_{\alpha} \rightarrow \delta_{e}$ in the weak-* topology and hence $t_{\alpha} * f \rightarrow \delta_{e} * f=f$ uniformly [5, 4.2F]. Thus $L^{1}(K) * C(K)$ is dense in $C(K)$. By the Cohen Factorization theorem [4, 32.22] there exist $h \in L^{1}(K), g \in C(K)$ so that $f=h * g$. Now

$$
\left\|t_{\alpha} * f-f\right\|_{u} \leqq\left\|t_{\alpha} * h-h\right\|_{1}\|g\|_{u} \longrightarrow 0
$$

and $t_{\alpha} * f \in \operatorname{Trig}(K)$ so Trig $(K)$ is uniformly dense in $C(K)$.

REMARKS 2.14. (a) For $U \in K^{\wedge}$ we define $\chi_{U}(x)=\operatorname{tr}\left(U_{x}\right)$. Then 
two finite-dimensional representations $U$ and $V$ of $K$ are equivalent if and only if their characters are the same, i.e., $\chi_{U}=\chi_{V}$.

(b) If $h \in C(K)$, then $x \rightarrow h\left(x * y * x^{\vee} * z\right)$ is continuous on $K$ for each $y, z \in K$; see [5, 3.1B, 3.1G].

We now generalize Well's character formula for compact groups $[4,27.54]$.

THEOREM 2.15. A nonzero continuous function $h$ on $K$ satisfies

$$
h(y) h(z)=\int_{K} h\left(x * y * x^{\vee} * z\right) d m(x)
$$

if and only if $h(x)=k_{U}^{-1} \chi_{U}(x)$ for some $U \in K^{\curlywedge}$.

Proof. We first show that $h=k_{U}^{-1} \chi_{U}$ satisfies equation (1). Let $U \in K^{\wedge}, d=d_{U}$ and $\left\{\zeta_{j}\right\}_{j=1}^{d}$ an orthonormal basis for $H_{U}$. By equation (1) of Theorem 2.2

$$
\begin{aligned}
\sum_{j=1}^{d} k_{U}^{-1}\left\langle U_{y} \zeta_{i}, \zeta_{j}\right\rangle\left\langle U_{z} \zeta_{k}, \zeta_{k}\right\rangle & =\int_{K} \sum_{j=1}^{d}\left\langle U_{x} U_{y} \zeta_{j}, \zeta_{k}\right\rangle\left\langle U_{x^{\vee}} U_{z} \zeta_{k}, \zeta_{j}\right\rangle d m(x) \\
& =\int_{K}\left\langle U_{x} U_{y} U_{x^{\vee}} U_{z} \zeta_{k}, \zeta_{k}\right\rangle d m(x) .
\end{aligned}
$$

Thus

$$
\begin{aligned}
k_{U}^{-1} \chi_{U}(y) \chi_{U}(z) & =\sum_{k=1}^{d} \sum_{j=1}^{d} k_{U}^{-1}\left\langle U_{y} \zeta_{j}, \zeta_{j}\right\rangle\left\langle U_{z} \zeta_{k}, \zeta_{k}\right\rangle \\
& =\int_{K} \operatorname{tr}\left(U_{x} U_{y} U_{x^{\vee}} U_{z}\right) d m(x)
\end{aligned}
$$

and a straightforward calculation shows that $\operatorname{tr}\left(U_{x} U_{y} U_{x^{v}} U_{z}\right)=$ $\chi_{U}\left(x * y * x^{2} * z\right)$ which implies (1) as desired.

Conversely, suppose $h$ satisfies (1). If $U_{h}=0$ for all $U \in K^{\wedge}$ then $0=\left\langle h, u_{j k}\right\rangle$ for all coordinate functions $u_{j k}$ and Corollary 2.10 implies $h=0$ contrary to hypothesis. Suppose $U$ in $K^{\wedge}$ satisfies $\int_{K} U_{x} h(x) d m(x) \neq 0$. Let $z \in K$ and $g=h^{z}$. Then

$$
h(z) U_{h}=\int_{K} \int_{K} g^{x}\left(x^{\vee} * y\right) U_{y} d m(y) d m^{\vee}(x) .
$$

So if $t \in K$ and $\zeta, \eta \in H_{U}$ then using [5, 5.1D] and Fubini's theorem repeatedly we have

$$
\begin{aligned}
\left\langle U_{t} h(z) U_{h} \xi, \eta\right\rangle & =\int_{K} \int_{K} g^{x}(y)\left\langle U_{t} U_{x} U_{y} \xi, \eta\right\rangle d m(y) d m(x) \\
& =\int_{K} \int_{K} g_{y}\left(t^{\vee} * x\right)\left\langle U_{x} U_{y} \xi, \eta\right\rangle d m(x) d m(y) \\
& =\int_{K} \int_{K} g^{x}\left(x^{\vee} * y\right)\left\langle U_{y} U_{t} \xi, \eta\right\rangle d m(y) d m(x)=\left\langle h(z) U_{h} U_{t} \xi, \eta\right\rangle .
\end{aligned}
$$


Since $U$ is irreducible we have $h(z) U_{h}$ is scalar for all $z \in K$. Since $h \neq 0, U_{h}$ is scalar, say $U_{h}=\alpha I$. Using equation (1) and [5, 5.1D].

$$
h(z) U_{h}=\int_{K} \int_{K} h(y) U_{x^{\vee}} U_{y} U_{z^{\vee}} U_{x} d m(y) d m(x)=U_{h} \int_{K} U_{x^{\vee}} U_{z^{\vee}} U_{x} d m(x)
$$

so, in particular, $h(e) I_{U}=\int_{K} U_{x^{\vee}} U_{x} d m(x)$. If $\xi \in H_{U}$ with $\|\xi\|=1$ then as in the proof of Theorem $2.2 d_{U} k_{U}^{-1}=\int_{K}\left\langle U_{x} \xi, U_{x} \xi\right\rangle d m(x)$ and hence $d_{U} k_{U}^{-1}=h(e)$. Now

$$
h(z) d_{U}=\operatorname{tr} \int_{K} U_{x^{\vee}} U_{z^{\vee}} U_{x} d m(x)=\operatorname{tr}\left(U_{z^{\vee}} \int_{K} U_{x^{\vee}} U_{x} d m(x)\right)=\chi_{U}\left(z^{\curlyvee}\right) k_{U}^{-1} d_{U},
$$

which implies $h(z)=k_{\bar{U}}^{-1} \chi_{\bar{U}}(z)$ where $\bar{U}$ is the conjugate representation of $U$. Since $\bar{U} \in K^{\wedge}$ the proof is complete.

3. Fourier transform. The development and notation in this section follows closely that found in Chapter 28 of [4]. We continue to assume $K$ is a compact hypergroup. The ${ }^{*}$-algebra $\Pi_{U \in K^{\wedge}} B\left(H_{U}\right)$ will be denoted by $\mathscr{E}\left(K^{\wedge}\right)$; scalar multiplication, addition, multiplication and the adjoint of an element are defined coordinatewise. Let $E=\left(E_{U}\right)$ be an element of $\mathscr{E}\left(K^{\wedge}\right)$. For $1 \leqq p<\infty$ we define

$$
\|E\|_{p}=\left(\sum_{U \in K^{\wedge}} k_{U}\left\|E_{U}\right\|_{\varphi_{p}}^{p}\right)^{1 / p} \text { and }\|E\|_{\infty}=\sup \left\{\left\|E_{U}\right\|_{\varphi_{\infty}}\right\} \text {. }
$$

The norms $\|\cdot\|_{\varphi_{p}}$ are the operator norms of $[4$, D. 37, D. 36(e)] and the notation $\mathscr{C}_{p}\left(K^{\wedge}\right), \mathscr{E}_{00}\left(K^{\wedge}\right)$ and $\mathscr{C}_{0}\left(K^{\wedge}\right)$ is as in $[4,28.24]$.

Definition 3.1. For $\mu \in M(K)$ let $\mu^{\wedge}(U)=\bar{U}_{\mu}$ for each $U \in K^{\wedge}$. Then $\mu^{\wedge} \in \mathscr{E}\left(K^{\wedge}\right)$ and is called a Fourier-Stieltjes transform of $\mu$. If $f \in L^{1}(K)$ then $f^{\wedge}(U)=\bar{U}_{f}$ and we call $f^{\wedge}$ a Fourier transform of $f$.

THEOREM 3.2. For each $\mu \in M(K)$ the mapping $\mu \rightarrow \mu^{\wedge}$ is a non norm-increasing *-isomorphism of the algebra $M(K)$ into the algebra $\mathscr{E}_{\infty}\left(K^{\wedge}\right)$.

Proof. Since $\bar{U} \in K^{\wedge}$ it is immediate that the map is a *-homomorphism and that $\left\|\mu^{\wedge}\right\|_{\infty} \leqq\|\mu\|$. If $\bar{U}_{\mu}=0$ for all $U \in K^{\wedge}$ then $\int_{K} u_{j k} d \mu=0$ for all coordinate functions $u_{j k}$. Thus the continuity of the map $f \rightarrow \int_{K} f d \mu$ and 2.13 imply $\int_{K} f d \mu=0$ for all $f \in C(K)$ so that $\mu=0$.

THEOREM 3.3. The map $f \rightarrow f^{\wedge}$ is a non norm-increasing ${ }^{*}$-isomorphism of $L^{1}(K)$ onto a dense subalgebra of $\mathscr{C}_{0}\left(K^{\wedge}\right)$. 
Proof. Imitate the proof in $[4,28.40]$.

THEOREM 3.4. The map $f \rightarrow f^{\wedge}$ is an inner product preserving map of $L^{2}(K)$ onto $\mathscr{E}_{2}\left(K^{\wedge}\right)$. In particular, $\left\|f^{\wedge}\right\|_{2}=\|f\|_{2}$. For $f \in$ $L^{2}(K)$ we have

$$
f=\sum_{U \in K^{\wedge}} k_{U} \sum_{j, k=1}^{d_{U}}\left\langle f^{\wedge}(U) \zeta_{k}^{U}, \zeta_{j}^{U}\right\rangle u_{j k}
$$

where the series converges in the $L^{2}$-norm.

Proof. Use Corollary 2.10.

The next theorem and its corollaries show that the notation of unitary representation is appropriate for a compact hypergroup precisely when the hypergroup is in fact a group. Also, these results generalize $[3,2.2]$ and $[8,3.1]$.

THeOREM 3.5. Let $K$ be a compact hypergroup, $U \in K^{\wedge}$ and $T$ a weak operator closed subgroup of the unitary operators on $H_{U}$. Then $S=\left\{x \in K: U_{x} \in T\right\}$ is a closed subhypergroup of $K$.

Proof. Clearly $e \in S$ and $S^{\smile}=S$. We need only show $S * S \subseteq S$. Let $x, y \in S$ and $\xi \in H_{U}$. Consider

$$
\langle\xi, \xi\rangle=\left\langle U_{x} U_{y} \xi, U_{x} U_{y} \xi\right\rangle=\int_{K}\left\langle U_{z} \xi, U_{x} U_{y} \xi\right\rangle d \delta_{x} * \delta_{y}(z)
$$

and note $\left|\left\langle U_{z} \xi, U_{x} U_{y} \xi\right\rangle\right| \leqq\langle\xi, \xi\rangle$. Also, the map $z \rightarrow\left\langle U_{z} \xi, U_{x} U_{y} \xi\right\rangle$ is continuous and the support of $\delta_{x} * \delta_{y}$ is compact so a straightforward argument shows that $\langle\xi, \xi\rangle=\left\langle U_{z} \xi, U_{t} \xi\right\rangle$ for all $\xi \in H_{U}, z, t \in$ support $\delta_{x} * \delta_{y}$. In particular, choosing $z=t$ it follows that $U_{z}$ is unitary for all $z$ in the support of $\delta_{x} * \delta_{y}$. Now if $z, t \in$ support $\delta_{x} * \delta_{y}$ then $\langle\xi, \xi\rangle=\left\langle U_{t^{\vee}} U_{z} \xi, \xi\right\rangle$ which implies $U$ is constant on the support of $\delta_{x} * \delta_{y}$, i.e., if $z \in$ support $\delta_{x} * \delta_{y}$

$$
U_{z}=\int_{K} U_{t} d \delta_{x} * \delta_{y}(t)=U_{x} U_{y} \in T
$$

Thus $S * S \subseteq S$.

CoRollary 3.6. If $K$ and $U$ are as in 3.5 then $S=\{x \in K$ : $\left.U_{x}=I\right\}$ is a closed subhypergroup of $K$.

CoROllaRY 3.7. Let $N=\bigcap_{U_{\in K^{\wedge}}}\left\{x \in K: U_{x}\right.$ is unitary $\}$. Then $N$ is the maximal subgroup of $K$. 
Proof. Let $M$ denote the maximal subgroup of $K$. If $x \in M$ then $U_{x} U_{x}^{*}=U_{e}=I$ so that $M \cong N$. Notice that $N$ is a closed subhypergroup of $K$ by Theorem 3.5. If $x \in N$ and $U \in K^{\wedge}$, we have $U_{\hat{o}_{x^{*} x^{\vee}}}=U_{x} U_{x^{\vee}}=I=U_{e}$ and hence $\left(\delta_{x} * \delta_{x}\right)=\delta_{e}$. Theorem $3.2 \mathrm{im}$ plies $\delta_{x} * \delta_{x^{\vee}}=\delta_{e}$ so that $x \in M$.

4. Functions with absolutely convergent Fourier series. In this section we define the Fourier-Stieltjes series of a measure and study in some detail the set $A(K)$ of those $L^{1}(K)$ functions with absolutely convergent Fourier series.

Definitions 4.1. Let $\mu \in M(K)$ and $U \in K^{\wedge}$. Set $A_{U}=\mu^{\wedge}(\bar{U})^{*}$ and write $A$ for the element $\left(A_{U}\right)$ of $\mathscr{E}\left(K^{\wedge}\right)$. The $A_{U}$ are called the coefficient operators of $\mu_{\text {and }}$ and formal expression $\sum_{U \in K^{\wedge}} k_{U} \operatorname{tr}\left(A_{U} U\right)$ is called the Fourier-Stieltjes series of $\mu$. If $\mu=f d m$ for some $f \in L^{1}(K)$ we call $\sum_{U \in K^{\wedge}} k_{U} \operatorname{tr}\left(A_{U} U\right)$ the Fourier series of $f$. If $f \in$ $L^{1}(K), f \approx \sum_{U \in K^{\wedge}} k_{U} \operatorname{tr}\left(A_{U} U\right)$ with $\sum_{U \in K^{\wedge}} k_{U}\left\|A_{U}\right\|_{\varphi_{1}}<\infty$ we say $f$ has an absolutely convergent Fourier series. For $f \in A(K)$ we define $\|f\|_{\varphi_{1}}=\left\|f^{\wedge}\right\|_{1}$.

Proposition 4.2. Let $f \in A(K), f \approx \sum_{U_{\epsilon} K^{\wedge}} k_{U} \operatorname{tr}\left(A_{U} U\right)$ Then $f$ is equal a.e., to the continuous function $\sum_{U \in K^{\wedge}} k_{U} \operatorname{tr}\left(A_{U} U_{x}\right)$ and so can be regarded as an element of $C(K)$. Also, $\|f\|_{U} \leqq\|f\|_{\varphi_{1}}$. Furthermore, the mapping $f \rightarrow f^{\wedge}$ is a norm-preserving linear isomorphism of $A(K)$ onto $\mathscr{E}_{1}\left(K^{\wedge}\right)$ and so $A(K)$ is a Banach space.

Proof. The proof here is similar to the group case [4, 34.5, $34.6,34.7]$.

We call a complex-valued function $f$ on $K$ positive-definite (p.d.) if $f$ is continuous and $0 \leqq \sum_{i=1}^{n} \sum_{j=1}^{n} a_{i} \bar{a}_{j} f\left(x_{i} * x_{j}^{\ulcorner}\right)$for each choice of complex numbers $a_{i}$ and elements $x_{i} \in K$. We denote the set of p.d. functions by $P(K)$.

LEMma 4.3. If $f \in P(K)$ then $\left\langle f^{\wedge}(U) \xi, \xi\right\rangle \geqq 0$ for all $U \in K^{\wedge}$ and $\xi \in H_{U}$. In particular, $\operatorname{tr}\left(f^{\wedge}(U)\right) \geqq 0$ for all $f \in P(K)$.

Proof. Clearly, we may assume $\|\xi\|=1$. Now extend $\xi$ to an orthonormal basis $\left\{\zeta_{j}\right\}$ for $H_{U}$ where $\xi=\zeta_{1}$. It follows that $\left\langle f^{\wedge}(U) \xi, \xi\right\rangle=\int_{K} u_{11} f d m$. However, $u_{11}=k_{L}^{1 / 2} u_{11} * k_{U}^{1 / 2} u_{11}^{*}$ which implies

$$
\left\langle f^{\wedge}(U) \xi, \xi\right\rangle=\int_{K} f d\left(k_{U}^{1 / 2} u_{11} m * k_{U}^{1 / 2} u_{11}^{*} m\right) \geqq 0
$$

where the last inequality follows from $[5,11.1 \mathrm{~A}, 11.1 \mathrm{~B}]$. 
The next theorem is instrumental in our characterization of $A(K)$. The proof given here applies Mercer's theorem following a method of Krein [6].

THEOREM 4.4. If $f \in P(K)$ then $f(e)=\|f\|_{u}=\sum_{U \in K^{\wedge}} k_{U} \operatorname{tr}\left(f^{\wedge}(U)\right)$ where the series converges absolutely.

Proof. [5, 11.1E] gives $f(e)=\|f\|_{u}$. Define $J(x, y)=f\left(y^{\prec} * x\right)$ which is continuous by $[5,3.1 \mathrm{~A}]$. Now define the operator $T_{J}$ : $L^{2}(K) \rightarrow L^{2}(K)$ by $T_{J}(g)(x)=\int_{K} J(x, y) g(y) d m(y)=g * f(x)$ for all $g \in$ $L^{2}(K)$. Since $T_{J}$ is just right convolution by $f, T_{J}$ is a bounded linear operator on $L^{2}(K)$ which is also compact [2, VI. 9.56]. Clearly $J(x, y)=\overline{J(y, x)}$ and $\left\langle T_{J} g, g\right\rangle \geqq 0$ since $f \in P(K)$. Thus $T_{J}$ satisfies the conditions of Mercer's theorem [2, XI. 8.57, XI. 8.58]. Therefore we may write $J(x, y)=\sum_{i=1}^{\infty} \lambda_{i} \Phi_{i}(x) \overline{\Phi_{i}(y)}$ where $\left\{\Phi_{i}\right\}_{i=1}^{\infty}$ is an orthonormal set of eigenfunctions for $T_{J}$ with corresponding eigenvalue $\lambda_{i}$ and the series converges absolutely and uniformly on $K \times K$. We have $\left\langle\Phi_{i}, f\right\rangle=\Phi_{i} * f(e)=\lambda_{i} \Phi_{i}(e)$ and $J(x, y)=f\left(y^{`} * x\right)$ so by setting $y=e$ we obtain

$$
f(x)=\sum_{i=1}^{\infty}\left\langle f, \Phi_{i}\right\rangle \Phi_{i}(x)
$$

with the series converging absolutely and uniformly on $K$. For $V \in K^{\wedge}$ the uniform convergence implies

$$
\left\langle f, v_{r s}\right\rangle=\sum_{i=1}^{\infty}\left\langle f, \Phi_{i}\right\rangle\left\langle\Phi_{i}, v_{r s}\right\rangle .
$$

Since $f, \Phi_{i} \in C(K)$ we have $f^{\wedge}, \Phi_{i}^{\wedge} \in \mathscr{E}_{2}\left(K^{\wedge}\right)$ (Theorem 3.4) so that $f^{\wedge} \Phi_{i}^{\wedge}=\lambda_{i} \Phi_{i}^{\wedge} \in \mathscr{E}_{1}\left(K^{\wedge}\right)$ Proposition 4.2 implies

$$
\Phi_{i}(x)=\sum_{U \in K^{\wedge}} k_{U} \operatorname{tr}\left(A_{U}\left(\Phi_{i}\right) U_{x}\right)
$$

with the series converging absolutely and uniformly. Thus

$$
f(x)=\sum_{j=1}^{\infty}\left\langle f, \Phi_{j}\right\rangle \sum_{U \in K^{\wedge}} k_{U} \operatorname{tr}\left(A_{U}\left(\Phi_{j}\right) U_{x}\right)
$$

and so by equation (1)

$$
\begin{aligned}
f(e) & =\sum_{j=1}^{\infty}\left\langle f, \Phi_{j}\right\rangle \sum_{U \in K^{\wedge}} k_{U} \operatorname{tr}\left(\Phi_{j}^{\wedge}(U)\right)=\sum_{U \in K^{\wedge}} \sum_{j=1}^{\infty} \sum_{k=1}^{d_{U}}\left\langle f, \Phi_{j}\right\rangle k_{U}\left\langle\Phi_{j}, u_{k k}\right\rangle \\
& =\sum_{U \in K^{\wedge}} k_{U} \operatorname{tr}\left(f^{\wedge}(U)\right) .
\end{aligned}
$$

Finally, Lemma 4.3 shows that the series $\sum_{U \in K^{\wedge}} k_{U} \operatorname{tr}\left(f^{\wedge}(U)\right)$ converges absolutely. 
LEMMA 4.5. Let $K$ be any locally compact hypergroup. If $f, g \in P(K)$ then $\bar{f} \in P(K)$ and $\alpha f+\beta g \in P(K)$ for all $\alpha, \beta \geqq 0$. Also, the pointwise limit of p.d. functions is p.d.

Proof. The only statement requiring proof here is the last one. Suppose $f_{n} \rightarrow f$ pointwise with $f_{n} \in P(K)$. By Theorem 4.4, $\left\|f_{n}\right\|_{n}=$ $f_{n}(e)$. A standard argument shows that $\sup \left\{\left\|f_{n}\right\|_{n}: n=1,2, \cdots\right\}<\infty$. Since 'support $\left(\delta_{x} * \delta_{y} v\right)$ is compact, the lemma follows easily by an application of Lebesgue's Dominated Convergence theorem.

THEOREM 4.6. $f \in P(K)$ if and only if $f \in A(K)$ and each $A_{U}$ is p.d. The condition each $A_{U}$ is p.d. is equivalent to each operator $f^{\wedge}(U)$ being p.d.

Proof. Sufficiency follows from Lemma 4.4 and an argument found in $[4,34.10]$. We assume $f \in P(K)$. Lemma 4.3 shows that $f^{\wedge}(U)$ is p.d. for each $U \in K^{\wedge}$. Moreover, $\operatorname{tr}\left(f^{\wedge}(U)\right)=\left\|f^{\wedge}(U)\right\|_{\varphi_{1}}$ $([4$, D.46]). By Theorem 4.4

$$
\sum_{U \in K^{\wedge}} k_{U}\left\|f^{\wedge}(U)\right\|_{\varphi_{1}}=\|f\|_{u}=f(e)<\infty
$$

and hence $f \in A(K)$.

THEOREM 4.7. $A(K)$ is precisely the linear span of $P(K)$. In fact, every $f \in P(K)$ has the form $f=f_{1}-f_{2}+i\left(f_{3}-f_{4}\right)$ where $f_{i} \in P(K)$.

Proof. This follows directly from Theorem 4.6 and [4, D.47].

THEOREM 4.8. If $f, g \in L^{2}(K)$ then $f * g \in A(K)$ and $\|f * g\|_{\varphi_{1}} \leqq$ $\|f\|_{2}\|g\|_{2}$.

Proof. Use Theorems 3.2, 3.4 and Hölder's inequality.

THEOREM 4.9. $A(K)=L^{2}(K) * L^{2}(K)$.

Proof. Apply 4.8 and mimic the argument in $[4,34.15]$.

The next theorem establishes regularity for $A(K)$; compare with $[1,2.9]$ and $[4,34.21]$.

THEOREM 4.10. Let $X, Y$ be disjoint, nonvoid, closed subsets of $K$. There is a function $f \in A(K)$ such that $f(X)=\{1\}, f(Y)=\{0\}$ and $f(K) \subseteq[0,1]$. 
Proof. Select a symmetric neighborhood $W$ of $e$ so that $W * W * X \leqq K-Y$. Let $f=m(W)^{-1} I_{W} * I_{W * X}$. Clearly $f$ is in $A(K)$ and it is not hard to show $f$ has the desired properties.

REMARKS 4.11. Since $\mathscr{E}_{1}\left(K^{\wedge}\right)$ is Banach algebra [4, 28.32(v)] it follows that $A(K)$ is a regular Banach algebra with convolution as multiplication and $\|\cdot\|_{\varphi_{1}}$ as norm. However, in contrast to the group case [4, 34.18], $A(K)$ may not form a Banach algebra under pointwise operations. In fact, we give an example of a finite abelian hypergroup where $A(K)$ fails to be a pointwise Banach algebra.

ExAMPLE 4.12. Let $K=\{e, a, b\}$ and $K^{\wedge}=\{1, \chi, \psi\}$ be as in $[5,9.1 \mathrm{C}]$. Since $\chi \in P(K)$ we have $\|\chi\|_{\varphi_{1}}=\chi(e)=1$ but $\left\|\chi^{2}\right\|_{\varphi_{1}}=$ $(666 / 612)>1$, i.e., $\left\|\chi^{2}\right\|_{\varphi_{1}}>\|\chi\|_{\varphi_{1}}\|\chi\|_{\varphi_{1}}$. The difficulty here is that the product of p.d. functions need not be p.d.

\section{REFERENCES}

1. A. Chilana and K. A. Ross, Spectral synthesis in hypergroups, to appear.

2. N. Dunford and J. T. Schwartz, Linear operators $I, I I$, Interscience Publishers Inc., 1958 and 1963.

3. C. F. Dunkl, The measure algebra of a locally compact hypergroup, Trans. Amer. Math. Soc., 179 (1973), 331-348.

4. E. Hewitt and K. A. Ross, Abstract Harmonic Analysis II, Springer-Verlag, 1970.

5. R. I. Jewett, Spaces with an abstract convolution of measures, Advances in Math., 18 (1975), 1-101.

6. M. G. Krein, Hermitian-positive kernels on homogeneous spaces, I and II, Ukrain. Mat. Z., 1 (1949), 64-98. English translation: Amer. Math. Soc. Translation, Ser. 2, Vol. 34 (1963), 69-164.

7. L. Nachbin, On the finite dimensionality of every irreducible representation of a compact group, Proc. Amer. Math. Soc., 12 (1961), 11-12.

8. K. A. Ross, Hypergroups and centers of measure algebras, Ist. Naz. Alta. Mat. (Symposia Math.), volume XXXII, to appear.

9. - Centers of hypergroups, Trans. Amer. Math. Soc., to appear.

10. R. Spector, Apercu de la theorie des hypergroupes, Lecture Notes in Mathematics \#497. (Analyse Harmonique sur les Groupes de Lie, Sem. Nancy-Strasbourg 1973-1975), Springer-Verlag.

Received November 19, 1977.

BUCKNELL UNIVERSITY

LEWISBURG, PA 17837 



\section{PACIFIC JOURNAL OF MATHEMATICS}

\section{EDITORS}

DONALD BABBITT (Managing Editor)

University of California

Los Angeles, California 90024

HUgo RossI

University of Utah

Salt Lake City, UT 84112

C. C. MOORE and ANDREW OGG

University of California

Berkeley, CA 94720

\section{J. DUGUNDJI}

Department of Mathematics University of Southern California Los Angeles, California 90007

R. Finn and J. Milgram Stanford University Stanford, California 94305

\section{ASSOCIATE EDITORS}

E. F. BECKENBACH

B. H. NEUMANN

F. WOLF

K. YoshidA

\section{SUPPORTING INSTITUTIONS}

UNIVERSITY OF BRITISH COLUMBIA CALIFORNIA INSTITUTE OF TECHNOLOGY UNIVERSITY OF CALIFORNIA MONTANA STATE UNIVERSITY UNIVERSITY OF NEVADA, RENO NEW MEXICO STATE UNIVERSITY OREGON STATE UNIVERSITY UNIVERSITY OF OREGON
UNIVERSITY OF SOUTHERN CALIFORNIA STANFORD UNIVERSITY UNIVERSITY OF HAWAII UNIVERSITY OF TOKYO UNIVERSITY OF UTAH WASHINGTON STATE UNIVERSITY UNIVERSITY OF WASHINGTON 


\section{Pacific Journal of Mathematics}

Vol. 85, No. $1 \quad$ September, 1979

Ralph Alexander, Metric averaging in Euclidean and Hilbert spaces...... 1

B. Aupetit, Une généralisation du théorème de Gleason-Kahane-Żelazko

pour les algèbres de Banach..............................

Lung O. Chung, Jiang Luh and Anthony N. Richoux, Derivations and

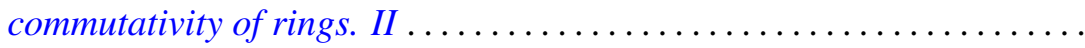

Lynn Harry Erbe, Integral comparison theorems for third order linear

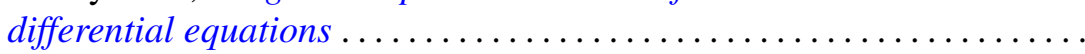

Robert William Gilmer, Jr. and Raymond Heitmann, The group of units of a

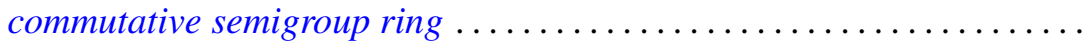

George Grätzer, Craig Robert Platt and George William Sands, Embedding lattices into lattices of ideals ...........................

Raymond D. Holmes and Anthony Charles Thompson, $n$-dimensional area and content in Minkowski spaces ....................... 77

Harvey Bayard Keynes and M. Sears, Modelling expansion in real flows....

Taw Pin Lim, Some classes of rings with involution satisfying the standard

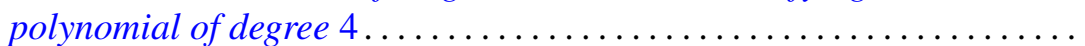

Garr S. Lystad and Albert Robert Stralka, Semilattices having bialgebraic congruence lattices ................................... 131

Theodore Mitchell, Invariant means and analytic actions . . . . . . . . . 145

Daniel M. Oberlin, Translation-invariant operators of weak type ........ 155

Raymond Moos Redheffer and Wolfgang V. Walter, Inequalities involving

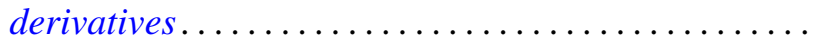

Eric Schechter, Stability conditions for nonlinear products and semigroups ................................

Jan Søreng, Symmetric shift registers ........................ 201

Toshiji Terada, On spaces whose Stone-Čech compactification is $\mathrm{Oz} \ldots \ldots .231$

Richard Vrem, Harmonic analysis on compact hypergroups ... 